OPEN ACCESS

Edited by:

Rosa M. Rivero,

Centro de Edafología y Biología Aplicada del Segura (CSIC), Spain

Reviewed by:

Qinghua Shi,

Shandong Agricultural University,

China

Guangxiao Yang,

Huazhong University of Science and Technology, China

*Correspondence:

Liang Chen

chenliang1034@126.com

†These authors have contributed equally to this work.

Specialty section:

This article was submitted to

Plant Abiotic Stress,

a section of the journal

Frontiers in Plant Science

Received: 03 September 2017 Accepted: 14 November 2017 Published: 28 November 2017

Citation:

Chen L, Fan J, Hu Z, Huang X, Amombo E, Liu A, Bi A, Chen K, Xie $Y$ and $F u J ~(2017)$ Melatonin Is Involved in Regulation

of Bermudagrass Growth and Development and Response to Low $K^{+}$Stress.

Front. Plant Sci. 8:2038.

doi: 10.3389/fpls.2017.02038

\section{Melatonin Is Involved in Regulation of Bermudagrass Growth and Development and Response to Low $\mathbf{K}^{+}$Stress}

\author{
Liang Chen ${ }^{1 * t}$, Jibiao Fan ${ }^{2 t}$, Zhengrong Hu ${ }^{1,3}$, Xuebing Huang ${ }^{1,3}$, Erick Amombo ${ }^{1,3}$, \\ Ao Liu ${ }^{1,3}$, Aoyue Bi ${ }^{1,3}, \mathrm{Ke} \mathrm{Chen}^{4}$, Yan $\mathrm{Xie}^{1}$ and Jinmin $\mathrm{Fu}^{1}$ \\ ${ }^{1}$ Key Laboratory of Plant Germplasm Enhancement and Specialty Agriculture, Wuhan Botanical Garden, Chinese Academy \\ of Sciences, Wuhan, China, ${ }^{2}$ College of Animal Science and Technology, Yangzhou University, Yangzhou, China, ${ }^{3}$ University \\ of Chinese Academy of Sciences, Beijing, China, ${ }^{4}$ College of Resources and Environmental Science, South-Central \\ University for Nationalities, Wuhan, China
}

Melatonin (N-acetyl-5-methoxytryptamine) plays critical roles in plant growth and development and during the response to multiple abiotic stresses. However, the roles of melatonin in plant response to $\mathrm{K}^{+}$deficiency remain largely unknown. In the present study, we observed that the endogenous melatonin contents in bermudagrass were remarkably increased by low $\mathrm{K}^{+}(\mathrm{LK})$ treatment, suggesting that melatonin was involved in bermudagrass response to LK stress. Further phenotype analysis revealed that exogenous melatonin application conferred Bermudagrass enhanced tolerance to LK stress. Interestingly, exogenous melatonin application also promoted bermudagrass growth and development at normal condition. Furthermore, the $\mathrm{K}^{+}$ contents measurement revealed that melatonin-treated plants accumulated more $\mathrm{K}^{+}$in both shoot (under both control and LK condition) and root tissues (under LK condition) compared with those of melatonin non-treated plants. Expression analysis indicated that the transcripts of $\mathrm{K}^{+}$transport genes were significantly induced by exogenous melatonin treatment in bermudagrass under both control and LK stress conditions, especially under a combined treatment of LK stress and melatonin, which may increase accumulation of $\mathrm{K}^{+}$content profoundly under $\mathrm{LK}$ stress and thereby contributed to the LK-tolerant phenotype. In addition, we investigated the role of melatonin in the regulation of photosystem II (PSII) activities under LK stress. The chlorophyll fluorescence transient (OJIP) curves were obviously higher in plants grown in LK with melatonin ( $L K+M e l)$ than those of plants grown in LK medium without melatonin application for 1 or 2 weeks, suggesting that melatonin plays important roles in PSII against LK stress. After a combined treatment of LK stress and melatonin, the values for performance indexes $\left(\mathrm{Pl}_{\mathrm{ABS}}, \mathrm{Pl}_{\text {Total }}\right.$, and $\left.\mathrm{PI}_{\mathrm{CS}}\right)$, flux ratios $\left(\varphi \mathrm{P}_{0}, \Psi \mathrm{E}_{0}\right.$, and $\left.\varphi \mathrm{E}_{0}\right)$ and specific energy fluxes $\left(E T_{O} / R C\right)$ were significantly improved compared with those of LK stress alone, suggesting that melatonin plays positive roles in protecting PSIl activity under LK stress. Collectively, this study reveals an important role of melatonin in regulating bermudagrass response to LK stress.

Keywords: bermudagrass, melatonin, LK stress, $\mathrm{K}^{+}$content, photosystem II (PSII) activity 


\section{INTRODUCTION}

Potassium is an essential element for plant growth and development, and a vital determinant of crop yield and quality. Potassium $\left(\mathrm{K}^{+}\right)$also plays crucial roles in many fundamental processes in living plant cells, such as osmoregulation, electrical neutralization, regulation of membrane potential, cotransport of sugars, and so on (Clarkson and Hanson, 1980; Xu et al., 2006; Wang and $\mathrm{Wu}, 2013)$. The concentration of $\mathrm{K}^{+}$in the cytoplasm is approximately $100 \mathrm{mM}$, while it varies from 10 to $200 \mathrm{mM}$ in the vacuoles (Walker et al., 1996). Although the $\mathrm{K}^{+}$concentration is high in plant cells, it is limited in soils, typically within the micromolar range $(0.1-1 \mathrm{mM})$ (Schroeder et al., 1994; Maathuis, 2009). Therefore, plants evolved a series of mechanisms, including physiological, biochemical, and morphological alterations, to respond to $\mathrm{K}^{+}$-deficiency stress (Schachtman and Shin, 2007; Wang and Wu, 2013; Wang et al., 2016).

Under low- $\mathrm{K}^{+}(\mathrm{LK})$ conditions, plants absorb $\mathrm{K}^{+}$through the high-affinity $\mathrm{K}^{+}$transporters and some $\mathrm{K}^{+}$channels (Maathuis and Sanders, 1997; Véry et al., 2014). In Arabidopsis, the AtHAK5 transporter (Rubio et al., 2000; Nieves-Cordones et al., 2010) and AKT1 channel (Lagarde et al., 1996; Hirsch et al., 1998; Spalding et al., 1999; Ivashikina et al., 2001) expressed primarily in the roots, were identified as the two major $\mathrm{K}^{+}$ uptake components, which mediate $\mathrm{K}^{+}$absorption in roots under LK conditions (Gierth et al., 2005; Pyo et al., 2010; Nieves-Cordones et al., 2014). AtHAK5 gene transcription was remarkably induced by $\mathrm{K}^{+}$deficiency, while AKT1 was regulated mainly at the posttranslational level (Gierth et al., 2005; Xu et al., 2006).

Melatonin ( $N$-acetyl-5-methoxytryptamine) was initially identified as an important animal hormone, which regulates sleep, mood, sexual behavior, circadian rhythms, and anti-oxidative activities (Tan et al., 2003; Reiter et al., 2009; Galano et al., 2011; Escames et al., 2012; Venegas et al., 2012; Calvo et al., 2013). Later studies revealed that melatonin is also ubiquitously presented in plants and unicellular organisms (Dubbels et al., 1995; Van Tassel et al., 1995; Hardeland and Poeggeler, 2003). Melatonin is widely involved in various aspects of plant growth and development, including flowering, circadian rhythms, leaf senescence, root system architecture (Arnao and Hernández-Ruiz, 2009; Byeon et al., 2012; Pelagio-Flores et al., 2012; Tan et al., 2012; Wang et al., 2013; Zhang et al., 2014). Tan et al. (2012) reported that the root system and production were greater in the melatonin-treated corn plants than those of the non-melatonin-treated plants (Tan et al., 2012). Likewise, melatonin promoted soybean growth and increased seed production (Wei et al., 2015). In addition, another important role of melatonin is related to abiotic and biotic stresses response, including salt, drought, extreme temperature, and pathogen infection (Zhang et al., 2013; Bajwa et al., 2014; Lee et al., 2014; Shi and Chan, 2014; Shi et al., 2015a,b,c). The role of melatonin involvement in LK stress response, however, remains largely unknown.

Potassium is a primary, essential nutrient for turfgrass production. A deficiency in $\mathrm{K}$ confers bermudagrass reduced winter hardiness, decreased disease resistance and diminished rhizome and stolon production (Miller, 1999). The present study revealed that the endogenous melatonin contents in bermudagrass were remarkably increased under LK condition. Further phenotype analysis revealed that exogenous melatonin application conferred Bermudagrass not only enhanced tolerance to LK stress, but also promoted bermudagrass growth and development under normal condition. Furthermore, melatonintreated plants accumulated more $\mathrm{K}^{+}$in both shoot (under both control and LK condition) and root tissues (under LK condition) compared with those of melatonin non-treated plants. Expression analysis indicated that the transcripts of $\mathrm{K}^{+}$transport genes were significantly induced by exogenous melatonin treatment in bermudagrass under both control and LK stress conditions, especially under a combined treatment of LK stress and melatonin. In addition, the role of melatonin in the regulation of photosystem II (PSII) activities under LK stress was further investigated. Collectively, this study reveals an important role of melatonin in regulating bermudagrass response to LK stress.

\section{MATERIALS AND METHODS}

\section{Plant Materials and Growth Conditions}

Bermudagrass 'WBD128' collected from Xiaojiang City, Zhejiang Province, China, was used in this study. Bermudagrass seeds were surface-sterilized with $75 \%$ ethanol for $1 \mathrm{~min}$ followed by $10 \% \mathrm{NaClO}$ for $3 \mathrm{~min}$ then washed with sterile water for four times. The sterilized bermudagrass seeds were sown on MS medium containing $1.5 \%$ sucrose, $\mathrm{pH}$ 5.8. After stratification at $4^{\circ} \mathrm{C}$ for 3 days in darkness, the MS plates were transferred to the growth chamber $\left(16 \mathrm{~h}\right.$ light $/ 8 \mathrm{~h}$ dark, $\left.26^{\circ} \mathrm{C}\right)$ for seed germination.

Uniform stolons of the genotype 'WBD128' were planted in plastic pots, which were kept in the greenhouse for 1 month to establish under growth conditions of $12 \mathrm{~h}$ light $/ 12 \mathrm{~h}$ dark, $28^{\circ} \mathrm{C}$.

\section{Treatment}

For LK stress treatment, 6-day-old bermudagrass plants on MS plate were transferred to LK or MS medium with different concentrations of melatonin and treated for the indicated times described in the figure legends section, and then the fresh weight and primary root length were recorded. The LK medium was modified from MS medium according to the method described previously ( $\mathrm{Xu}$ et al., 2006). Briefly, $2.99 \mathrm{mM} \mathrm{CaCl} 2$ and $1.25 \mathrm{mM} \mathrm{KH}_{2} \mathrm{PO}_{4}$ were replaced by $2.99 \mathrm{mM} \mathrm{Ca}\left(\mathrm{NO}_{3}\right)_{2}$ and $1.25 \mathrm{mM} \mathrm{NH}_{4} \mathrm{H}_{2} \mathrm{PO}_{4}$, $18.79 \mathrm{mM} \mathrm{KNO}_{3}$ and $20.6 \mathrm{mM} \mathrm{NH}_{4} \mathrm{NO}_{3}$ were removed, and $1.5 \mathrm{mM} \mathrm{MgSO}_{4}$ was unchanged. The final $\mathrm{K}^{+}$concentration in $\mathrm{LK}$ agar medium was adjusted by adding $\mathrm{KCl}$. In the present study, the $\mathrm{K}^{+}$concentration was $100 \mu \mathrm{M}$ in the $\mathrm{LK}$ medium.

For analysis of low $\mathrm{K}^{+}$stress on the PSII activities of bermudagrass, the established 'WBD128' plants were transferred to LK or MS liquid medium with or without melatonin for the indicated times described in figure legends. 


\section{Chlorophyll (Chl) a Fluorescence Transient}

The pulse-amplitude modulation (PAM) fluorometer (PAM 2500, Heinz Walz GmbH) was employed to measure chlorophyll (Chl) a fluorescence transient. The measurement was performed as described previously (Chen et al., 2014; Hu et al., 2016). After the collected bermudagrass leaves were pre-adapted in the dark for $30 \mathrm{~min}$, the OJIP transients were triggered by red light $\left(3,000 \mu \mathrm{mol}\right.$ photons $\left.\mathrm{m}^{-2} \mathrm{~s}^{-1}\right)$ to guarantee closure of all reaction centers of PS II and to evaluate a real fluorescence intensity. The $\mathrm{Chl}$ a fluorescence emission induced by the strong light pulses was subsequently tested and digitized between $10 \mu \mathrm{s}$ and $320 \mathrm{~ms}$. The OJIP transient data was processed using the JIP-test as reported by Chen et al. (2014).

\section{Quantification of Melatonin}

The measurement of endogenous melatonin contents was performed by enzyme-linked immunosorbent assay (ELISA) as previously described with some modifications (Fan et al., 2015; $\mathrm{Hu}$ et al., 2016). Briefly, a $0.3 \mathrm{~g}$ of leaf tissues was ground into fine powder in liquid nitrogen, transferred into tubes containing $5 \mathrm{ml}$ of extraction solution (Acetone:Methanol:Water $=89: 10: 1$ ), and then homogenized on ice for $1 \mathrm{~h}$. After centrifugation at $4500 \times g$ for $5 \mathrm{~min}$ at $4^{\circ} \mathrm{C}$, the supernatant was transferred to a new tube containing $0.5 \mathrm{ml}$ of $1 \%$ trichloric acid. The mixture was centrifuged at $4500 \times \mathrm{g}$ for $10 \mathrm{~min}$ at $4^{\circ} \mathrm{C}$, and the extract was used for quantification of endogenous melatonin using the Melatonin ELISA Kit (EK-DSM; Buhlmann Laboratories AG, Schonenbuch, Switzerland) according to the manufacturer's instruction.

\section{Measurement of $\mathrm{K}^{+}$Content}

After growth on MS or LK medium for 2 weeks, shoots and roots of the bermudagrass seedlings were harvested for $\mathrm{K}^{+}$content measurements. The plant samples were washed thoroughly with distilled water, placed in oven at $105^{\circ} \mathrm{C}$ for $30 \mathrm{~min}$ to deactivate the enzymes, and then dried at $80^{\circ} \mathrm{C}$ for $48 \mathrm{~h}$. After weighing, the samples were ground into a fine power, and then digested in a solution $\left(\mathrm{HClO}_{4}: \mathrm{HNO}_{3}=1: 4, \mathrm{v} / \mathrm{v}\right)$. Subsequently, the $\mathrm{K}^{+}$ content assays were performed using ICP-MS (Thermo Fisher Scientific, Waltham, MA, United States) as previously described (Xie et al., 2017).

\section{RNA Isolation and Quantitative Real-Time PCR}

The total RNA was isolated from the bermudagrass roots using TRIzol reagent (Invitrogen, United States) according to the manufacturer's instruction. DNaseI was used to remove any contaminated genomic DNA, and then a $2 \mu \mathrm{g}$ of total RNA was reversely transcribed into cDNAs using M-MLV reverse transcriptase (Promega, United States). Real-time quantitative RT-PCR was performed by the method described previously by Chen et al. (2012), and the CdACT2 gene was used as a quantitative control. The specific primer sequences were listed in Table 1.

\section{Statistical Analysis}

All experiments were performed with at least three repetitions. The significance of differences was determined by ANOVA or Student's $t$-test $(P<0.05)$, as indicated in the figure legends.

\section{RESULTS}

\section{The Endogenous Melatonin Contents under LK Stress}

To investigate the role of melatonin in plants response to LK stress, the endogenous melatonin production in bermudagrass leaves was measured after being treated with LK stress for 12 , 24 , and $72 \mathrm{~h}$. The results revealed that the endogenous melatonin contents were remarkably increased for 2- to 4.7 -fold during the 12-72 h of LK treatment (Figure 1). The significant increase of endogenous melatonin content by LK stress suggests that melatonin may be involved in plant response to LK stress in bermudagrass.

\section{Melatonin Involvement in Plant Growth and Development and LK Stress Response}

To further investigate the possible role of melatonin involvement in plant growth and development and LK stress response, we examined the phenotypic changes of bermudagrass under normal (MS) and LK stress conditions with or without melatonin treatment. The 6-day-old seedlings were transferred and vertically cultured on MS or LK medium containing 20 or $50 \mu \mathrm{m}$ melatonin for 1 week. It was found that under both MS and LK conditions, melatonin-treated plants grew better as characterized by greater fresh weight and higher root length compared with those of non-melatonin-treated plants (Figures 2A-D). The primary root length of 20 or $50 \mu \mathrm{m}$ melatonin-treated plants (MS+20 or $50 \mu \mathrm{m}$ melatonin) was 130 and $125 \%$ of those of non-treated plants, while under LK condition, the root length of melatonin-treated plants were increased to 156 and $158 \%$ of those of non-treated plants (Figures 2A-D), suggesting that melatonin was involved in regulating both plant growth and development and LK response. In addition, the $\mathrm{K}^{+}$contents of bermudagrass plants were measured after they were grown on MS or LK medium with or without $50 \mu \mathrm{m}$ melatonin application. Under LK condition, melatonin-treated plants accumulated more

\begin{tabular}{|c|c|}
\hline Primer name & Primer sequence $\left(5^{\prime}\right.$ to $\left.3^{\prime}\right)$ \\
\hline CdACT2 F & 5' - TCTGAAGGGTAAGTAGAGTAG -3' \\
\hline CdACT2 R & 5'- ACTCAGCACATTCCAGCAGAT -3' \\
\hline CdHAK9 F & 5' - TTCGTGAGGCTGGACGCGTCG -3' \\
\hline CdHAK9 R & 5' - CTGCCGTTCGTGGCGCGCACC -3' \\
\hline Potassium transporter 1 (CdKT1) F & 5' - TACGAGTCGTCGGTGGACGGG -3' \\
\hline Potassium transporter 1 (CdKT1) R & 5' - ATCTGGAACCGGACCCTGCGC -3' \\
\hline Potassium transporter 23 (CdKT23) F & 5'- GACGACTCGGCCGCGGGCGCG -3 \\
\hline Potassium transporter 23 (CdKT23) R & 5'- GCCGTAGACAACACCGAGGGT -3' \\
\hline
\end{tabular}




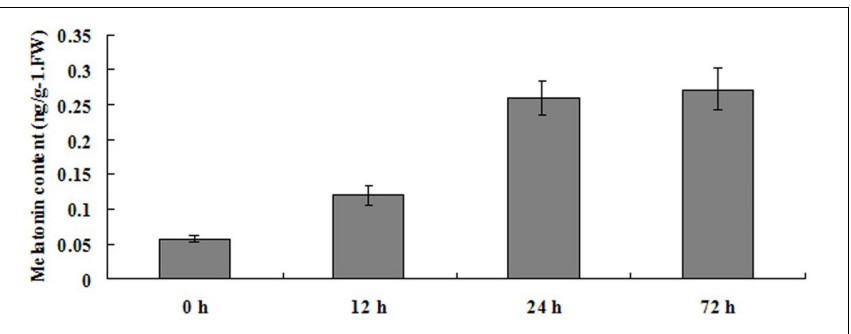

FIGURE 1 | Changes of endogenous melatonin concentration in response to LK stress in Bermudagrass. Six-day-old bermudagrass plants on MS plate were transferred to LK medium for $0,12,24$, and $72 \mathrm{~h}$. The experiments were repeated three times. Data shown are the mean $\pm \mathrm{SD}$.

$\mathrm{K}^{+}$in both shoot and root tissues compared with those of melatonin non-treated plants. By contrast, when plants were grown on MS medium, the higher $\mathrm{K}^{+}$accumulation in the shoots of melatonin-treated plants were observed compared with those of melatonin non-treated plants, whereas there was no significant difference in $\mathrm{K}^{+}$content in roots between melatonin and non-melatonin treated plants (Figure 3 ). The results suggest that the greater $\mathrm{K}^{+}$content accumulation in melatonin-treated plants may contribute to the LK-tolerant phenotype and improved growth and development of bermudagrass.

Next, the expression of genes involved in potassium transport was investigated by RT-PCR. The results revealed that expression levels of the three tested gene were significantly induced by LK stress or melatonin treatment, and further increased to higher levels after LK stress and melatonin combined treatment (Figure 4). These results suggested that the higher $\mathrm{K}^{+}$content accumulation in melatonin-treated plants may result from the increased expression of $\mathrm{K}^{+}$transport genes.

\section{Effects of Low $\mathrm{K}^{+}$Stress on the Photosystem II (PSII) Activities of Bermudagrass}

To investigate whether melatonin is involved in regulation of PSII activities under LK stress, OJIP fluorescence transient curves were tested. As shown in Figure 5, the OJIP fluorescence transients were significantly affected by LK stress. The OJIP curves dropped remarkably after LK treatment compared with those of plants that were grown in control condition (MS liquid medium). There were no significant differences in the OJIP transient curves between plants grown in MS medium with or without melatonin application for 1 week or 2 weeks. However, the OJIP curves were obviously higher in plants grown in LK with melatonin $(\mathrm{LK}+\mathrm{Mel})$ than those of plants grown in LK medium without melatonin application for 1 or 2 weeks (Figure 5), suggesting that melatonin played important roles in PSII against LK stress. Besides, the OJIP curves of plants grown in LK medium for 2 weeks were significantly lower than those of plants grown in LK medium for 1 week, revealing that PSII activity was further decreased during plants exposure to LK stress for prolonged days.

The JIP-test was further applied to study the photosynthetic behavior of PSII. The results showed that the photosynthetic behavior of PSII was significantly different between plants treated with or without melatonin under low $\mathrm{K}^{+}$stress, but not under control condition (MS) with or without exogenous melatonin (Figures 6-8). In brief, the structural and functional parameters of photosynthetic behavior including performance indexes, flux ratios and specific energy fluxes were improved in melatonintreated plants under LK condition.

The performance indexes (PI) such as $\mathrm{PI}_{\mathrm{ABS}}, \mathrm{PI}_{\text {Total }}$, and $\mathrm{PI}_{\mathrm{CS}}$ are widely used to describe the overall activity of PSII. As shown in Figure 6, the values of $\mathrm{PI}_{\mathrm{ABS}}, \mathrm{PI}_{\mathrm{Total}}$, and $\mathrm{PI}_{\mathrm{CS}}$ were all significantly lower in plants treated by LK stress than those of plants grown under MS condition. For instance, the value of $\mathrm{PI}_{\mathrm{ABS}}$ reduced remarkably after $\mathrm{LK}$ treatment for 1 week, and it was further decreased after LK for 2 weeks. However, it was reversed by melatonin application during LK treatment (Figure 6). Similar results were also observed in the parameters, such as $\mathrm{PI}_{\text {Total }}$ and $\mathrm{PI}_{\mathrm{CS}}$ (Figure 6). These results suggested that exogenous melatonin played a protective role in PSII activity under LK stress.

For the flux ratios, $\varphi \mathrm{P}_{0}$ (maximum quantum yield of primary photochemistry) decreased significantly under LK stress, and it further declined as the LK treatment time prolonged. After melatonin application during LK stress, the parameter of $\varphi \mathrm{P}_{0}$ was higher than that of LK treatment (Figure 7). Similarly, the parameters of other flux ratios such as $\Psi E_{0}$ (Efficiency/probability with which a PSII trapped electron is transferred from $\mathrm{Q}_{\mathrm{A}}$ to $\mathrm{Q}_{\mathrm{B}}$ ) and $\varphi \mathrm{E}_{0}$ (quantum yield of the electron transport flux from $\mathrm{Q}_{\mathrm{A}}$ to $\mathrm{Q}_{\mathrm{B}}$ ) displayed the alteration pattern similar to $\varphi \mathrm{P}_{0}$ (Figure 7 ). These results imply that melatonin plays positive roles in improving the behavior in both electron donor side and acceptor side of PSII when plants were treated by LK stress. The parameters of specific energy fluxes, including $\mathrm{ET}_{\mathrm{O}} / \mathrm{RC}$ [Electron transport flux (further than $\mathrm{QA}^{-}$) per $\mathrm{RC}$ ] and $\mathrm{DI}_{\mathrm{O}} / \mathrm{RC}$ (Energy fluxes for dissipation) were further calculated. The $\mathrm{ET}_{\mathrm{O}} / \mathrm{RC}$ value was lower in the plants of LK treatment than that of MS treatment, and a slight but not significant increase was observed in the plants treated with melatonin when the plants were treated for 1 week (Figure 8). After the plants were treated for 2 weeks, a remarkable decrease of $\mathrm{ET}_{\mathrm{O}} / \mathrm{RC}$ was observed in the plants by $\mathrm{LK}$ treatment, and melatonin mitigated the damage effectively (Figure 8). On the contrary, $\mathrm{DI}_{\mathrm{O}} / \mathrm{RC}$ increased slightly in the plants after they were treated by LK stress for 1 week. When the plants were treated for 2 weeks, $\mathrm{DI}_{\mathrm{O}} / \mathrm{RC}$ showed the highest value under $\mathrm{LK}$ condition among four different treatments, while $\mathrm{DI}_{\mathrm{O}} / \mathrm{RC}$ decreased in LK+Mel regime (Figure 8).

\section{DISCUSSION}

Recently, several studies have revealed that the functions of melatonin are related to many aspects of plant growth and development. The most frequently mentioned functions of melatonin are its roles in abiotic and biotic stresses such as drought (Shi et al., 2015a; Wei et al., 2015), salt (Wei et al., 2015), extreme temperature (Bajwa et al., 2014; Shi and Chan, 2014; Shi et al., 2015b,c), and bacterial infection (Lee et al., 2014; Shi et al., 2015b). However, so far little is known about the roles of 

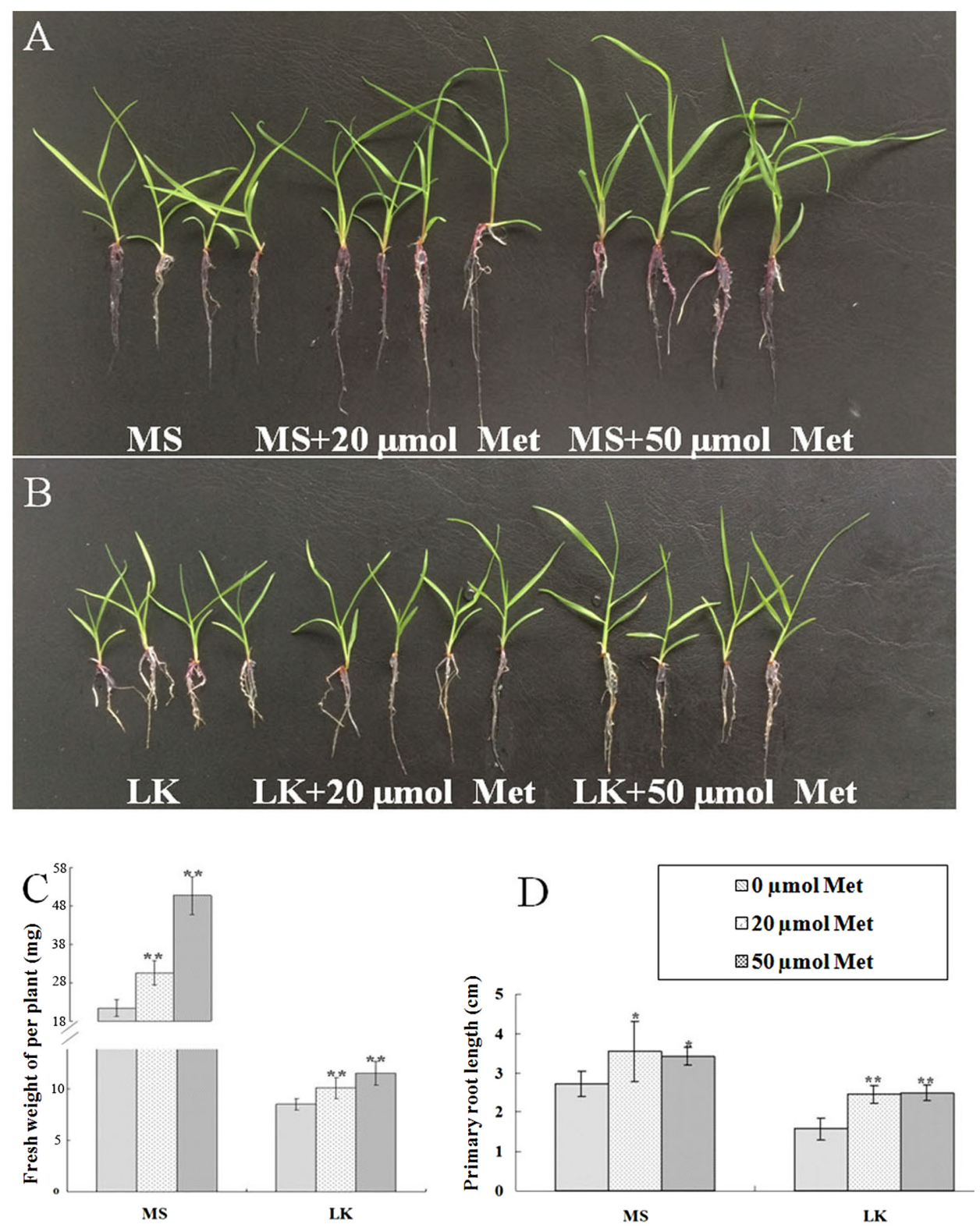

FIGURE 2 | Exogenous melatonin application promoted growth and development of bermudagrass and conferred bermudagrass improved tolerance to LK stress. Six-day-old bermudagrass plants were transferred to MS (A) or LK agar plates (B) with different concentrations of melatonin for another 2 weeks. (C) Quantification of the fresh weight and (D) primary root length as shown in (A) and (B). Data shown are the mean $\pm \mathrm{SD}(n=3)$. ${ }^{* *} P<0.01$, ${ }^{*} P<0.05$ by Student's $t$-test.

melatonin involved in LK stress response. Only one recent study reported that melatonin was involved in the absorption of $\mathrm{K}^{+}$ elements by plants under stress conditions (Li et al., 2016).

In this study, we revealed at least three important issues. Firstly, exogenous melatonin application promoted bermudagrass growth and development. Wei et al. (2015) demonstrated that melatonin has significant potential for enhancement of soybean growth and seed production. Similarly, the root system was larger in melatonin-treated corn plants than that of non-melatonin-treated corn plants, and thus the estimated production of the melatonin-treated corn was $20 \%$ greater than that from the non-melatonin-treated plants (Tan et al., 2012). Consistent with previous investigations, we found that primary root length and fresh weight were larger in melatonin-treated bermudagrass plants than those of non-melatonin-treated plants.

Secondly, the endogenous melatonin contents were remarkably increased after LK treatment, and exogenous melatonin application conferred bermudagrass improved LK stress tolerance. Previous studies have reported that the endogenous melatonin contents were increased when plants were exposed to abiotic stress, such as cold and heat (Shi 


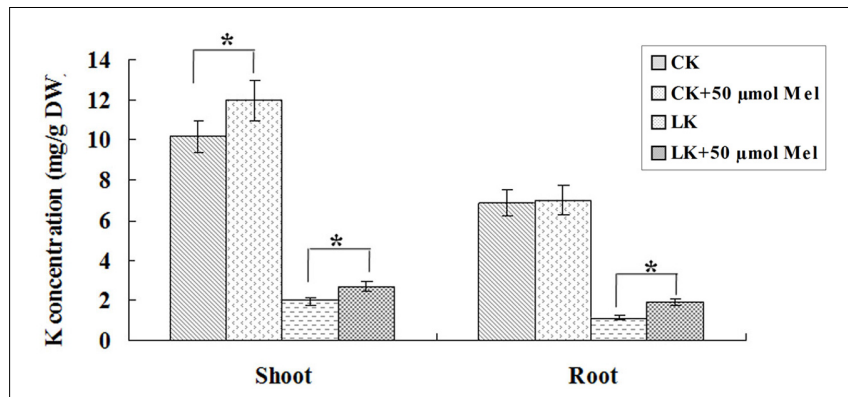

FIGURE 3 | Effects of melatonin treatment on $\mathrm{K}^{+}$contents in roots and shoots under MS or LK stress. The $\mathrm{K}^{+}$content was measured after the plants were transferred to MS or LK medium with or without $50 \mu \mathrm{m}$ melatonin. Data shown are the mean $\pm \operatorname{SD}(n=3) .{ }^{*} P<0.05$ by Student's $t$-test.

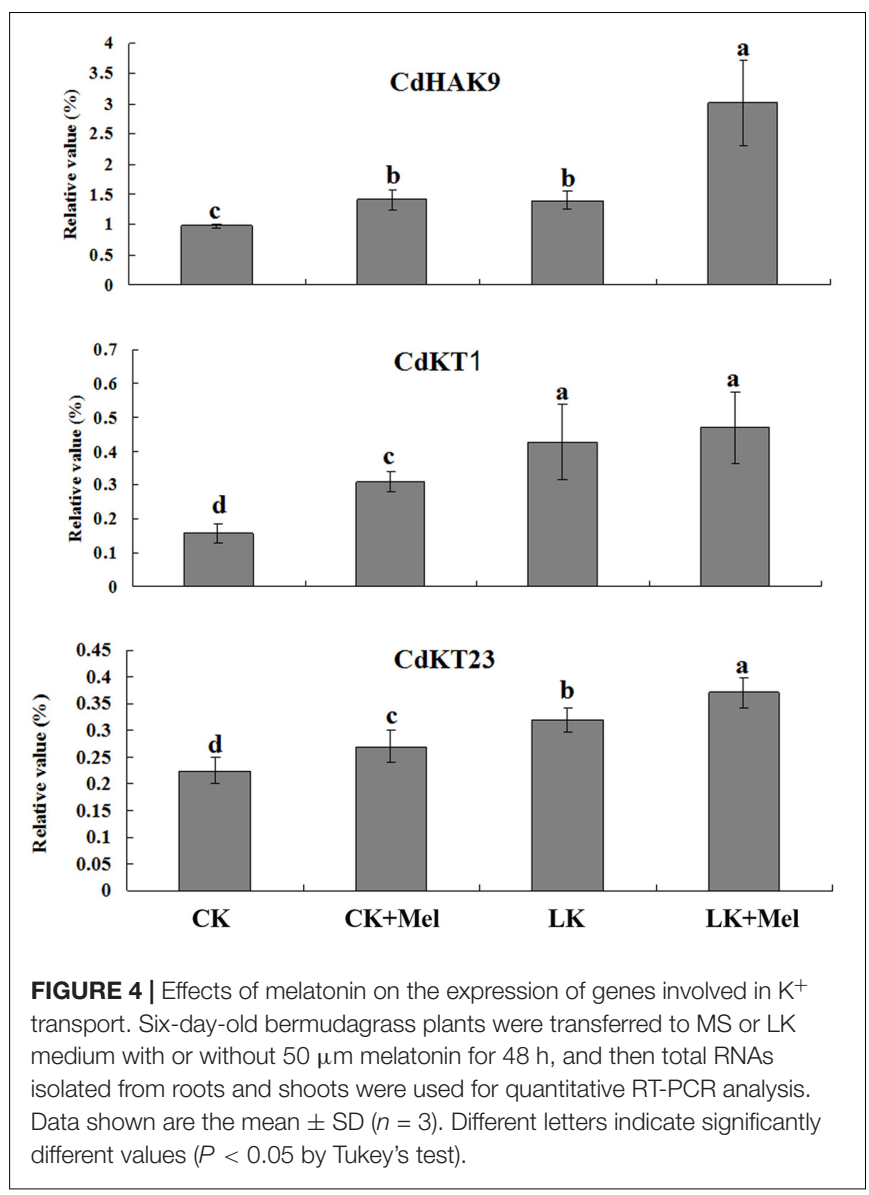

and Chan, 2014; Shi et al., 2015c). Therefore, we investigated whether endogenous melatonin concentration was enhanced in LK-treated plants. Meaningfully, our results revealed that the endogenous melatonin contents in bermudagrass were remarkably increased after LK treatment, suggesting the possible involvement of melatonin in plant response to LK stress in bermudagrass. Furthermore, exogenous melatonin application conferred bermudagrass improved LK stress tolerance. The measurement of $\mathrm{K}^{+}$contents and expression analysis of $\mathrm{K}^{+}$transport genes revealed that under $\mathrm{LK}$

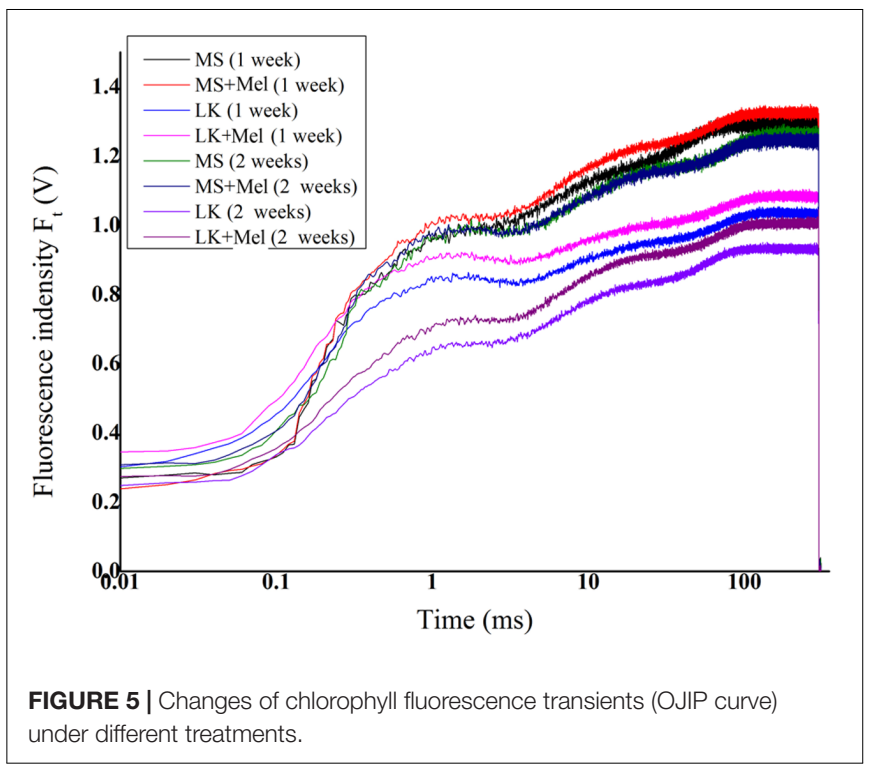

stress, the melatonin-treated plants increased expression of $\mathrm{K}^{+}$transport genes, resulted in the greater $\mathrm{K}^{+}$content accumulation, and thereby contributed to the LK-tolerant phenotype. Next step, it is meaningful to investigate how the melatonin regulates the expression of $\mathrm{K}^{+}$transport genes.

Thirdly, melatonin is involved in regulation of PSII activities under LK stress. Chlorophyll $a$ fluorescence has been widely employed to investigate plant photosystem activity especially under abiotic stress conditions, and it can be as an indicator to evaluate the ability of plant tolerance to abiotic stress (Chen et al., 2014; Fan et al., 2015; Hu et al., 2016). As an essential nutrient element, $\mathrm{K}^{+}$significantly affects many fundamental processes, including photosynthesis and assimilation product transport or translocation, and thereby determines plant growth and development (Wang et al., 2013). However, little is known in detail of how LK stress regulates bermudagrass PSII activity, and whether melatonin is involved in regulation of PSII activities under LK stress. In the present study, our results revealed that the OJIP curves dropped remarkably after LK treatment compared with those of plants grown under control condition, revealing that the LK stress resulted in the damage to bermudagrass PSII. By contrast, the OJIP curves were obviously increased in plants grown in LK medium with melatonin application $(\mathrm{LK}+\mathrm{Mel})$, suggesting that melatonin plays important roles in PSII against LK stress. Our previous studies also revealed that melatonin plays essential roles in PSII against cold stress (Fan et al., 2015; $\mathrm{Hu}$ et al., 2016). These results consistently implied the crucial roles of melatonin involvement in PSII regulation under abiotic stress.

The JIP-test was further employed to quantify the photosynthetic behavior of plants based on structural and functional parameters. The performance index PI total, which represented energy conservation from exciton to the reduction of PSI end acceptors, incorporated several parameters from OJIP, and thus is a sensitive parameter of the JIP-test for assessing 

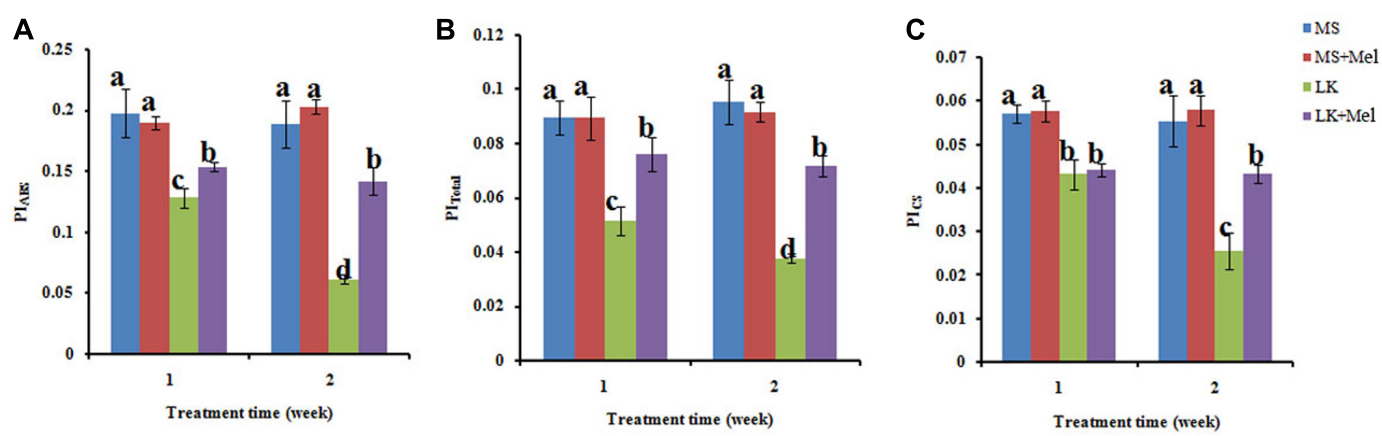

FIGURE 6 | Changes of performance index (PI) as deduced by JIP-test analysis of fluorescence transients. (A) $\mathrm{Pl}$ ABS, $\mathrm{PI}$ for energy conservation from exciton to the reduction of the intersystem electron; (B) PI $\mathrm{Ttal}_{\text {, }} \mathrm{PI}$ for energy conservation from exciton to the reduction of $\mathrm{PSI}$ end acceptors; (C) $\mathrm{Pl}$ CS, $\mathrm{PI}$ on a cross section basis. Data shown are the mean $\pm \mathrm{SD}(n=3)$. Different letters indicate significantly different values $(P<0.05$ by Tukey's test).
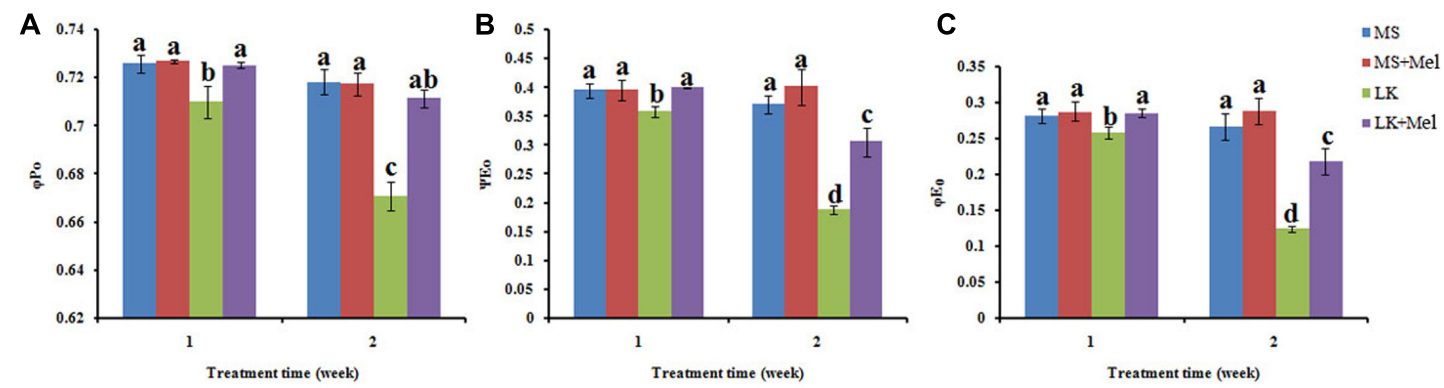

FIGURE 7 | Changes of flux ratios as deduced by JIP-test analysis of fluorescence transients. (A) $\varphi P_{0}$, the maximum quantum yield of PSII photochemistry; (B) $\Psi E_{0}$, efficiency/probability with which a PSIl trapped electron is transferred from $Q_{A}$ to $Q_{B} ;(\mathbf{C}) \varphi E_{0}$, the quantum yield of the electron transport flux from $Q_{A}$ to $Q_{B}$. Data shown are the mean $\pm \mathrm{SD}(n=3)$. Different letters indicate significantly different values $(P<0.05$ by Tukey's test).
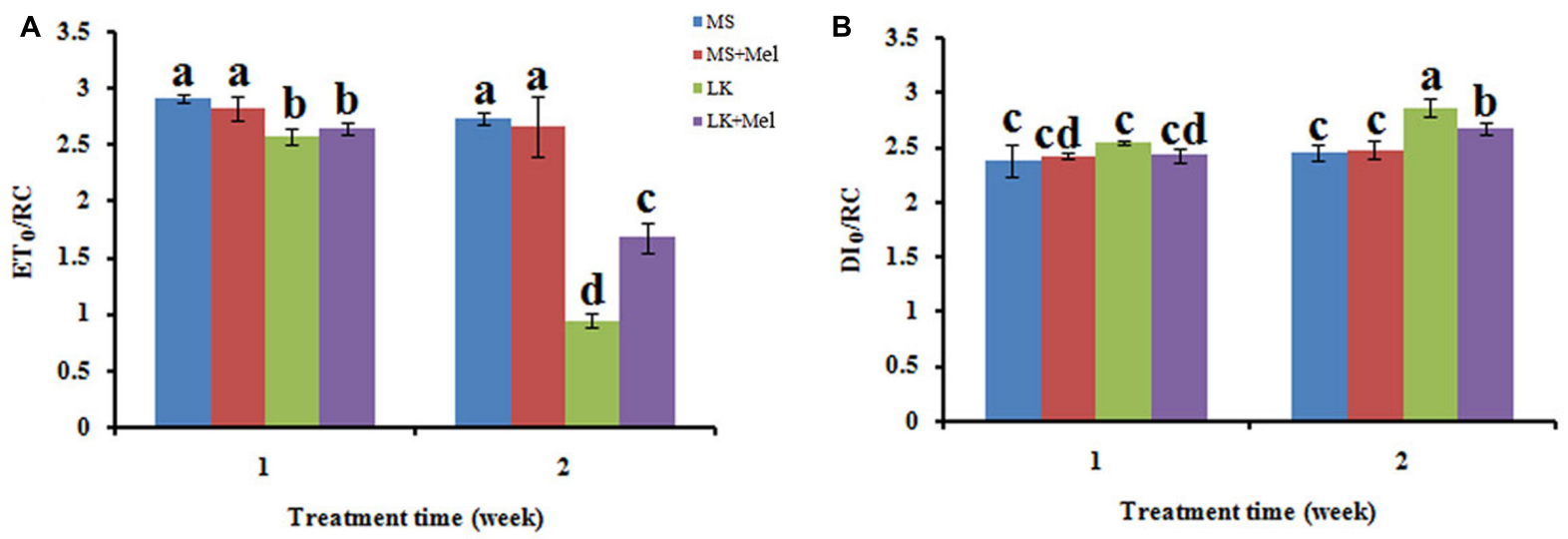

FIGURE 8 | Changes of specific energy fluxes per active PSIl reaction center (RC) as deduced by JIP-test analysis of fluorescence transients. (A) ET /RC, electron transport flux (further than $\mathrm{QA}^{-}$) per $\mathrm{RC}$; (B) $\mathrm{Dl}_{\mathrm{O}} / \mathrm{RC}$, energy fluxes for dissipation.

plant photochemical activities under abiotic stress condition (Clark et al., 2000; Yusuf et al., 2010; Chen et al., 2013; Liu et al., 2016). Here it was found that melatonin greatly improved the $\mathrm{PI}_{\text {Total }}$ value under LK stress, suggesting that melatonin plays positive roles in maintaining the photochemical activities under LK stress. The flux ratios, such as $\varphi \mathrm{P}_{0}, \varphi \mathrm{E}_{0}$, and $\Psi \mathrm{E}_{0}$ could evaluate the degree of injury of PSII components. In the present study, LK stress significantly decreased the behavior of $\varphi \mathrm{P}_{0}, \varphi \mathrm{E}_{0}$, and $\Psi \mathrm{E}_{0}$, revealing the damage to PSII caused by $L K$ stress. After melatonin application during LK stress, these parameters were significantly improved compared with those of LK stress, implying that melatonin plays positive roles in improving the 
behavior in both electron donor side and acceptor side of PSII under LK stress.

\section{CONCLUSION}

Our study showed that LK stress can result in the enhancement of endogenous melatonin content in bermudagrass. Furthermore, exogenous melatonin application not only promoted bermudagrass growth and development under normal condition but also conferred bermudagrass enhanced tolerance to LK stress. The enhanced LK stress tolerance by melatonin can be attributed to longer primary root, greater $\mathrm{K}^{+}$content accumulation due to improved expression of $\mathrm{K}^{+}$transport genes and increased PSII activities.

\section{REFERENCES}

Arnao, M. B., and Hernández-Ruiz, J. (2009). Protective effect of melatonin against chlorophyll degradation during the senescence of barley leaves. J. Pineal Res. 46, 58-63. doi: 10.1111/j.1600-079X.2008.00625.x

Bajwa, V. S., Shukla, M. R., Sherif, S. M., Murch, S. J., and Saxena, P. K. (2014). Role of melatonin in alleviating cold stress in Arabidopsis thaliana. J. Pineal Res. 56, 238-245. doi: 10.1111/jpi.12115

Byeon, Y., Park, S., Kim, Y. S., Park, D. H., Lee, S., and Back, K. (2012). Lightregulated melatonin biosynthesis in rice during the senescence process in detached leaves. J. Pineal Res. 53, 107-111. doi: 10.1111/j.1600-079X.2012. 00976.x

Calvo, J. R., González-Yanes, C., and Maldonado, M. D. (2013). The role of melatonin in the cells of the innate immunity: a review. J. Pineal Res. 55, 103-120. doi: 10.1111/jpi.12075

Chen, K., Chen, L., Fan, J., and Fu, J. (2013). Alleviation of heat damage to photosystem II by nitric oxide in tall fescue. Photosynth. Res. 116, 21-31. doi: 10.1007/s11120-013-9883-5

Chen, K., Sun, X., Amombo, E., Zhu, Q., Zhao, Z., Chen, L., et al. (2014). High correlation between thermotolerance and photosystem II activity in tall fescue. Photosynth. Res. 122, 305-314. doi: 10.1007/s11120-014-0035-3

Chen, L., Ren, F., Zhou, L., Wang, Q. Q., Zhong, H., and Li, X. B. (2012). The Brassica napus calcineurin B-Like 1/CBL-interacting protein kinase 6 (CBL1/CIPK6) component is involved in the plant response to abiotic stress and ABA signalling. J. Exp. Bot. 63, 6211-6222. doi: 10.1093/jxb/ers273

Clark, A. J., Landolt, W., Bucher, J. B., and Strasser, R. J. (2000). Beech (Fagus sylvatica) response to ozone exposure assessed with a chlorophyll a fluorescence performance index. Environ. Pollut. 109, 501-507. doi: 10.1016/S0269-7491(00) 00053- 1

Clarkson, D. T., and Hanson, J. B. (1980). The mineral nutrition of higher plants. Annu. Rev. Plant Physiol. 31, 239-298. doi: 10.1146/annurev.pp.31.060180. 001323

Dubbels, R., Reiter, R. J., Klenke, E., Goebel, A., Schnakenberg, E., Ehlers, C., et al. (1995). Melatonin in edible plants identified by radioimmunoassay and by high performance liquid chromatography-mass spectrometry. J. Pineal Res. 18, 28-31. doi: 10.1111/j.1600-079X.1995.tb00136.x

Escames, G., Ozturk, G., Baño-Otálora, B., Pozo, M. J., Madrid, J. A., Reiter, R. J., et al. (2012). Exercise and melatonin in humans: reciprocal benefits. J. Pineal Res. 52, 1-11. doi: 10.1111/j.1600-079X.2011.00924.x

Fan, J., Hu, Z., Xie, Y., Chan, Z., Chen, K., Amombo, E., et al. (2015). Alleviation of cold damage to photosystem II and metabolisms by melatonin in Bermudagrass. Front. Plant Sci. 6:925. doi: 10.3389/fpls.2015.00925

Galano, A., Tan, D. X., and Reiter, R. J. (2011). Melatonin as a natural ally against oxidative stress: a physicochemical examination. J. Pineal Res. 51, 1-16. doi: 10.1111/j.1600-079X.2011.00916.x

Gierth, M., Mäser, P., and Schroeder, J. I. (2005). The potassium transporter AtHAK5 functions in $\mathrm{K}^{+}$deprivation-induced high-affinity $\mathrm{K}^{+}$uptake and

\section{AUTHOR CONTRIBUTIONS}

LC designed the research. LC, JFa, ZH, XH, AL, and $\mathrm{AB}$ performed experiments and data analysis. LC wrote the manuscript in close collaboration with all authors. $\mathrm{EA}, \mathrm{YX}, \mathrm{JFu}$, and $\mathrm{KC}$ revised the manuscript. All authors contributed to numerous discussions and revised the manuscript.

\section{FUNDING}

This research was supported by the National Natural Science Foundation of China (31401915 and 3127 2194).

AKT1 $\mathrm{K}^{+}$channel contribution to $\mathrm{K}^{+}$uptake kinetics in Arabidopsis roots. Plant Physiol. 137, 1105-1114. doi: 10.1104/pp.104.057216

Hardeland, R., and Poeggeler, B. (2003). Non-vertebrate melatonin. J. Pineal Res. 34, 233-241. doi: 10.1034/j.1600-079X.2003.00040.x

Hirsch, R. E., Lewis, B. D., Spalding, E. P., and Sussman, M. R. (1998). A role for the AKT1 potassium channel in plant nutrition. Science 280, 918-921. doi: $10.1126 /$ science. 280.5365 .918

Hu, Z., Fan, J., Chen, K., Amombo, E., Chen, L., and Fu, J. (2016). Effects of ethylene on photosystem II and antioxidant enzyme activity in Bermuda grass under low temperature. Photosynth. Res. 128, 59-72. doi: 10.1007/s11120-015-0199-5

Ivashikina, N., Becker, D., Ache, P., Meyerhoff, O., Felle, H. H., and Hedrich, R. (2001). $\mathrm{K}^{+}$channel profile and electrical properties of Arabidopsis root hairs. FEBS Lett. 508, 463-469. doi: 10.1016/S0014-5793(01)03114-3

Lagarde, D., Basset, M., Lepetit, M., Conejero, G., Gaymard, F., Astruc, S., et al. (1996). Tissue-specific expression of Arabidopsis AKT1 gene is consistent with a role in $\mathrm{K}^{+}$nutrition. Plant J. 9, 195-203. doi: 10.1046/j.1365-313X.1996. 09020195.x

Lee, H. Y., Byeon, Y., and Back, K. (2014). Melatonin as a signal molecule triggering defense responses against pathogen attack in Arabidopsis and tobacco. J. Pineal Res. 57, 262-268. doi: 10.1111/jpi.12165

Li, C., Liang, B., Chang, C., Wei, Z., Zhou, S., and Ma, F. (2016). Exogenous melatonin improved potassium content in Malus under different stress conditions. J. Pineal Res. 61, 218-229. doi: 10.1111/jpi.12342

Liu, A., Hu, Z., Bi, A., Fan, J., Gitau, M. M., Amombo, E., et al. (2016). Photosynthesis, antioxidant system and gene expression of bermudagrass in response to low temperature and salt stress. Ecotoxicology 25, 1445-1457. doi: 10.1007/s10646-016-1696-9

Maathuis, F. J. M. (2009). Physiological functions of mineral macronutrients. Curr. Opin. Plant Biol. 12, 250-258. doi: 10.1016/j.pbi.2009.04.003

Maathuis, F. J. M., and Sanders, D. (1997). Regulation of $\mathrm{K}^{+}$absorption in plant root cells by external $\mathrm{K}^{+}$: interplay of different plasma membrane $\mathrm{K}^{+}$ transporters. J. Exp. Bot. 48, 451-458. doi: 10.1093/jxb/48.Special_Issue.451

Miller, G. L. (1999). Potassium application reduces calcium and magnesium levels in Bermudagrass leaf tissue and soil. Hortscience 34, 265-268.

Nieves-Cordones, M., Alemán, F., Martínez, V., and Rubio, F. (2010). The Arabidopsis thaliana HAK5 $\mathrm{K}^{+}$transporter is required for plant growth and $\mathrm{K}^{+}$acquisition from low $\mathrm{K}^{+}$solutions under saline conditions. Mol. Plant 3, 326-333. doi: $10.1093 / \mathrm{mp} / \mathrm{ssp} 102$

Nieves-Cordones, M., Alemán, F., Martínez, V., and Rubio, F. (2014). K ${ }^{+}$uptake in plant roots. The systems involved, their regulation and parallels in other organisms. J. Plant Physiol. 171, 688-695. doi: 10.1016/j.jplph.2013.09.021

Pelagio-Flores, R., Muñoz-Parra, E., Ortiz-Castro, R., and López-Bucio, J. (2012). Melatonin regulates Arabidopsis root system architecture likely acting independently of auxin signaling. J. Pineal Res. 53, 279-288. doi: 10.1111/j. 1600-079X.2012.00996.X

Pyo, Y. J., Gierth, M., Schroeder, J. I., and Cho, M. H. (2010). High-affinity $\mathrm{K}^{+}$transport in Arabidopsis: AtHAK5 and AKT1 are vital for seedling 
establishment and postgermination growth under low-potassium conditions. Plant Physiol. 153, 863-875. doi: 10.1104/pp.110.154369

Reiter, R. J., Tan, D. X., Manchester, L. C., Paredes, S. D., Mayo, J. C., and Sainz, R. M. (2009). Melatonin and reproduction revisited. Biol. Reprod. 81, 445-456. doi: 10.1095/biolreprod.108.075655

Rubio, F., Santa-María, G. E., and Rodríguez-Navarro, A. (2000). Cloning of Arabidopsis and barley cDNAs encoding HAK potassium transporters in root and shoot cells. Physiol. Plant 109, 34-43. doi: 10.1034/j.1399-3054.2000. 100106.x

Schachtman, D. P., and Shin, R. (2007). Nutrient sensing and signaling: NPKS. Annu. Rev. Plant Biol. 58, 47-69. doi: 10.1146/annurev.arplant.58.032806. 103750

Schroeder, J. I., Ward, J. M., and Gassmann, W. (1994). Perspectives on the physiology and structure of inward-rectifying $\mathrm{K}^{+}$channels in higher plants: biophysical implications for K uptake. Annu. Rev. Biophys. Biomol. Struct. 23, 441-471. doi: 10.1146/annurev.bb.23.060194.002301

Shi, H., and Chan, Z. (2014). The cysteine2/histidine2-type transcription factor ZINC FINGER OF ARABIDOPSIS THALIANA 6-ACTIVATED C-REPEATBINDING FACTOR pathway is essential for melatonin-mediated freezing stress resistance in Arabidopsis. J. Pineal Res. 57, 185-191. doi: 10.1111/jpi.12155

Shi, H., Jiang, C., Ye, T., Tan, D. X., Reiter, R. J., and Zhang, H. (2015a). Comparative physiological, metabolomic, and transcriptomic analyses reveal mechanisms of improved abiotic stress resistance in bermudagrass [Cynodon dactylon (L). Pers.] by exogenous melatonin. J. Exp. Bot. 66, 681-694. doi: $10.1093 / \mathrm{jxb} / \mathrm{eru} 373$

Shi, H., Qian, Y., Tan, D. X., Reiter, R. J., and He, C. (2015b). Melatonin induces the transcripts of $C B F / D R E B 1 s$ and their involvement in both abiotic and biotic stresses in Arabidopsis. J. Pineal Res. 59, 334-342. doi: 10.1111/jpi.12262

Shi, H., Tan, D. X., Reiter, R. J., Ye, T., Yang, F., and Chan, Z. (2015c). Melatonin induces class A1 heat-shock factors (HSFA1s) and their possible involvement of thermotolerance in Arabidopsis. J. Pineal Res. 58, 335-342. doi: 10.1111/jpi. 12219

Spalding, E. P., Hirsch, R. E., Lewis, D. R., Sussman, M. R., and Lewis, B. D. (1999). Potassium uptake supporting plant growth in the absence of AKT1 channel activity. J. Gen. Physiol. 113, 909-918. doi: 10.1085/jgp.113.6.909

Tan, D. X., Hardeland, R., Manchester, L. C., Korkmaz, A., Ma, S., RosalesCorral, S., et al. (2012). Functional roles of melatonin in plants, and perspectives in nutritional and agricultural science. J. Exp. Bot. 63, 577-597. doi: 10.1093/ jxb/err256

Tan, D. X., Hardeland, R., Manchester, L. C., Poeggeler, B., Lopez-Burillo, S., Mayo, J. C., et al. (2003). Mechanistic and comparative studies of melatonin and classic antioxidants in terms of their interactions with the ABTS cation radical. J. Pineal Res. 34, 249-259. doi: 10.1034/j.1600-079X.2003.00037.x

Van Tassel, D. L., Roberts, N. J., and Oenill, S. D. (1995). Melatonin from higher plants-Isolation and identification of N-acetyl 5-methoxytryptamine. Plant Physiol. 108, 101.

Venegas, C., García, J. A., Escames, G., Ortiz, F., López, A., Doerrier, C., et al. (2012). Extrapineal melatonin: analysis of its subcellular distribution and daily fluctuations. J. Pineal Res. 52, 217-227. doi: 10.1111/j.1600-079X.2011.00931.x

Véry, A. A., Nieves-Cordones, M., Daly, M., Khan, I., Fizames, C., and Sentenac, H. (2014). Molecular biology of $\mathrm{K}^{+}$transport across the plant cell membrane: what do we learn from comparison between plant species? J Plant Physiol. 171, 748-769. doi: 10.1016/j.jplph.2014.01.011

Walker, D. J., Leigh, R. A., and Miller, A. J. (1996). Potassium homeostasis in vacuolate plant cells. Proc. Natl. Acad. Sci. U.S.A. 93, 10510-10514. doi: 10.1073/ pnas.93.19.10510

Wang, P., Sun, X., Li, C., Wei, Z., Liang, D., and Ma, F. (2013). Long-term exogenous application of melatonin delays drought-induced leaf senescence in apple. J. Pineal Res. 54, 292-302. doi: 10.1111/jpi.12017

Wang, X. P., Chen, L. M., Liu, W. X., Shen, L. K., Wang, F. L., Zhou, Y., et al. (2016). AtKC1 and CIPK23 synergistically modulate AKT1-mediated low potassium stress responses in Arabidopsis. Plant Physiol. 170, 2264-2277. doi: 10.1104/pp. 15.01493

Wang, Y., and Wu, W. H. (2013). Potassium transport and signaling in higher plants. Annu. Rev. Plant Biol. 64, 451-476. doi: 10.1146/annurev-arplant050312-120153

Wei, W., Li, Q. T., Chu, Y. N., Reiter, R. J., Yu, X. M., Zhu, D. H., et al. (2015). Melatonin enhances plant growth and abiotic stress tolerance in soybean plants. J. Exp. Bot. 66, 695-707. doi: 10.1093/jxb/eru392

Xie, Y., Han, S., Li, X., Amombo, E., and Fu, J. (2017). Ameliorates of salt stress on bermudagrass by the fungus Aspergillus aculeatus. Mol. Plant Microbe Interact. 30, 245-254. doi: 10.1094/MPMI-12-16-0263-R

Xu, J., Li, H. D., Chen, L. Q., Wang, Y., Liu, L. L., He, L., et al. (2006). A protein kinase, interacting with two calcineurin B-like proteins, regulates $\mathrm{K}^{+}$ transporter AKT1 in Arabidopsis. Cell 125, 1347-1360. doi: 10.1016/j.cell.2006. 06.011

Yusuf, M. A., Kumar, D., Rajwanshi, R., Strasser, R. J., Tsimilli-Michael, M., Govindjee, et al. (2010). Overexpression of $\gamma$-tocopherol methyl transferase gene in transgenic Brassica juncea plants alleviates abiotic stress: physiological and chlorophyll a fluorescence measurements. Biochim. Biophys. Acta 1797, 1428-1438. doi: 10.1016/j.bbabio.2010. 02.002

Zhang, N., Zhang, H. J., Zhao, B., Sun, Q. Q., Cao, Y. Y., Li, R., et al. (2014). The RNA-seq approach to discriminate gene expression profiles in response to melatonin on cucumber lateral root formation. J. Pineal Res. 56, 39-50. doi: 10.1111/jpi.12095

Zhang, N., Zhao, B., Zhang, H. J., Weeda, S., Yang, C., Yang, Z. C., et al. (2013). Melatonin promotes water-stress tolerance, lateral root formation, and seed germination in cucumber (Cucumis sativus L.). J Pineal Res. 54, 15-23. doi: 10.1111/j.1600-079X.2012.01015.x

Conflict of Interest Statement: The authors declare that the research was conducted in the absence of any commercial or financial relationships that could be construed as a potential conflict of interest.

Copyright $\odot 2017$ Chen, Fan, Hu, Huang, Amombo, Liu, Bi, Chen, Xie and Fu. This is an open-access article distributed under the terms of the Creative Commons Attribution License (CC BY). The use, distribution or reproduction in other forums is permitted, provided the original author(s) or licensor are credited and that the original publication in this journal is cited, in accordance with accepted academic practice. No use, distribution or reproduction is permitted which does not comply with these terms. 